\title{
Analytical Analysis of Multiscale Entropy of Time Series: White Noise and $1 / f$ Noise
}

\author{
Wei Han ${ }^{1,2}$, Yanwen Zhuo ${ }^{1}$, Chi Tang ${ }^{1}$, Wenbo Liu ${ }^{3}$, Shengyi Zhou ${ }^{1}$, Shuo Liư ${ }^{1}$, \\ Zhou Fei ${ }^{3}$ and Kangning $\mathrm{Xie}^{1^{*}}$ \\ ${ }^{1}$ School of Biomedical Engineering, Air Force Medical University, Xi'an 710000, Shaanxi, China; \\ ${ }^{2}$ Department of Medical Engineering, the 987th Hospital of Joint Logistics Support Force, Baoji \\ 721000, Shaanxi, China;
}

${ }^{3}$ Department of Neurosurgery, Xijing Hospital, Airforce Medical University, Xi'an 710000, Shaanxi, China

${ }^{*}$ Corresponding author

Keywords: Multiscale entropy, Time series, White noise, 1/f noise.

\begin{abstract}
Multiscale entropy (MSE) has attracted many applications in revealing the complexity of bio-signals since its introduction in early 2000. To better understand the MSE method, a lot of researchers have been working on its analytical solution. The existing analytical solutions are limited either to bulky double integrals, or to a proportional relation. This study intends to solve the double integrals and further approximate the analytical formula of MSE. Combining Costa's method with Briggs' integral and Taylor series expansion, we approximated the analytical formula of MSE for uncorrelated (white) noise. With the additional help of the eigenvalue of a fractal Brownian motion process, we approximated the analytical formula of MSE for correlated noise (1/f noise). The analytical formulae were verified on simulated power noise with known scaling exponent. The proposed analytical solution may shed some light on the understanding of MSE in analyzing biological time series.
\end{abstract}

\section{Introduction}

It has attracted considerable attentions to quantify the "complexity" of physiologic signals in the attempt to distinguish different physiological conditions, e.g., between health and disease, or between the young and the elderly [1, 2]. Many measures, e.g., approximate entropy and sample entropy, have been employed to quantify complexity. Nevertheless, these entropy-based measures quantify complexity of time series based on a single time scale so that they miss the features associated with signal structure [3]. To solve the problem, Costa et al have proposed a new measure, multiscale entropy (MSE) to identify complexity in biological systems [4, 5] by means of calculating sample entropy in multiple scales of the coarse-grained times series of the original one, which can obtain the dynamic features related to multiple scales. Since its introduction, the MSE method has become a prevailing method to quantify the complexity of signals in a variety of research fields, e.g., biomedicine [4, 6-8], finance [9], seismology [10], traffic [11] and spatial analysis [12].

To gain the insight of the MSE method, Costa et al obtained the analytical solution of MSE for two special cases: white noise and 1/f noise as double integrals (integral of error function, which is also integrals), which were later used to produce analytical solutions [5]. Further analytical analysis on MSE was carried out by Gao et al, who showed MSE method can reveal fundamental bi-scaling law [13]. In addition to the scale factor that is normally considered in MSE, they reported that with this additional phase space scale manipulation, different signals were better distinguished. However, Gao's analytical formula showed direct proportional relation between MSE values and an expression, rather than presenting an equation.

In this work, we report a new analytical solution of MSE by approximating the double integrals proposed by Costa et al. Then, the solution is evaluated to test its validity and the dependences of MSE on some parameters are discussed. 


\section{Preliminaries}

Costa et al have provided detailed analytical deviations of MSE for two special cases, uncorrelated and correlated noises with Gaussian distributions. In this section, we briefly introduce their idea and notations.

First, for uncorrelated Gaussian noise, one can observe $n$ data points, $x_{1}, x_{2}, \cdots, x_{n}$. Consider that each data point comes from uncorrelated stochastic variables $X$. Costa et al calculated the sample entropy $S_{E}(X)$,

$S_{E}=-\ln P_{r}\left(\left|x_{j}-x_{i}\right| \leq r\right)$

where $P_{r}$ is the probability that the distance between two data points of the coarse-grained time series (with scale $\tau$ ) is less than or equal to a predefined threshold $r$. This probability was shown as the following double-integral form:

$$
\begin{aligned}
& P_{r}\left(\left|x_{j}^{s}-x_{i}^{s}\right| \leq r\right) \\
& =\frac{\sqrt{s}}{2 \sigma} \sqrt{\frac{1}{2 \pi}} \int_{-\infty}^{\infty}\left\{\operatorname{erf}\left(\frac{x+r}{\sigma \sqrt{2 / s}}\right)-\operatorname{erf}\left(\frac{x-r}{\sigma \sqrt{2 / s}}\right)\right\} e^{-x^{2} s / 2 \sigma^{2}} d x
\end{aligned}
$$

where erf refers to the error function $\operatorname{erf}(x)=\frac{2}{\pi} \int_{0}^{x} e^{-u^{2}} d u$, and $\sigma$ is the standard deviation of the original time series of scale 1 , and $r$ is the threshold, which is defined as $r=c \sigma$, where $c$ is the tolerance factor, a constant typically between 0.15 and 0.3 .

Next, Costa analyzed the MSE for 1/f noise by mapping the correlated noise into white noise via orthogonal transformation. The correlated noise is assumed to come from a fractal Gaussian noise (fGn) process. It has a time-independent autocorrelation function depending only on lag $\tau$ and Hurst exponent $H$ [14]. Consider $n$ random variables $X_{i}(i=1,2, \cdots n)$. There is an orthogonal transform matrix $U^{T}$ (formed with eigenvectors $U_{i}$ of the covariance matrix of $X_{i}$ ) which can transform correlated $X_{i}$ into uncorrelated (independent) $Y_{i}$ with Gaussian distribution with standard deviation of $\sigma_{Y i}=\sqrt{\lambda_{i}}$, where $\lambda_{i}$ is the eigenvalues corresponding to the $U_{i}$.

\section{Proposed Analytical Analysis}

The above analytical MSE evaluations contain double integrals, making them difficult to shed light on the deeper mechanism. In this section, we propose analytical equations without integrals, which is straightforward and calculation efficient. A series of methods are involved including Briggs' integral, Taylor series expansion and eigenvalue calculation of a fractal Brownian process.

\section{For White Noise}

Data points of white noise are uncorrelated Gaussian. In order to analytically analyze equation (1), we note that Briggs [15] has given the integral of erf

$$
\int_{-\infty}^{\infty} e^{-(\alpha x+\beta)^{2}} \operatorname{erf}(\gamma x+\delta) d x=\frac{\sqrt{\pi}}{\alpha} \operatorname{erf}\left(\frac{\alpha \delta-\beta \gamma}{\sqrt{\alpha^{2}+\gamma^{2}}}\right)
$$

Thus, we can assume

$\gamma=\frac{1}{\sigma \sqrt{2 / s}}, \delta=\frac{r}{\sigma \sqrt{2 / s}}$ (first half of equation(1)), $\delta=\frac{-r}{\sigma \sqrt{2 / s}}$ (second half of equation (1)), $\alpha=\frac{\sqrt{s}}{\sqrt{2} \sigma}$, $\beta=0$

Equation (1) can then be easily reduced to $P_{r}\left(\left|x_{j}^{s}-x_{i}^{s}\right| \leq r\right)=\operatorname{erf}\left(\frac{\sqrt{s} r}{2 \sigma}\right)$

MSE for each scale $\tau$ is now

$\operatorname{MSE}(s)=-\ln \left(P_{r}\left(\left|x_{j}^{s}-x_{i}^{s}\right| \leq r\right)\right)$

$=-\ln \left(\operatorname{erf}\left(\frac{\sqrt{s} r}{2 \sigma}\right)\right)$

Since $r=c \sigma$, equation (3) becomes

$M S E(s)=-\ln \left(\operatorname{erf}\left(\frac{\sqrt{s c}}{2}\right)\right)$ 
Recall that, $\operatorname{erf}(x)$ has its Taylor expansion

$\operatorname{erf}(x)=\frac{2}{\sqrt{\pi}}\left(x-\frac{x^{3}}{3}+\frac{x^{5}}{10}+\cdots\right)$

We can see that, for $x<1$, erf $(x)$ can be estimated as the first term in the above equation, as $\operatorname{erf}(x) \approx \frac{2}{\sqrt{\pi}} x$. For typical settings in [5], $1 \leq s \leq 20,0<c<=0.5, x=\frac{\sqrt{s} c}{2}<1$, therefore, equation (4) can be approximated as

$$
\begin{aligned}
& \operatorname{MSE}(s, c)=-\ln \left(\operatorname{erf}\left(\frac{\sqrt{s} C}{2}\right)\right) \\
& \left.\approx-\ln \left(\frac{2}{\sqrt{\pi}} \frac{\sqrt{s} c}{2}\right)\right)=-\ln \left(\frac{\sqrt{s} C}{\sqrt{\pi}}\right)=-\ln \frac{c}{\sqrt{\pi}}-\frac{1}{2} \ln s
\end{aligned}
$$

If we keep the first two terms of erf $(x)$, as $\operatorname{erf}(x)=\frac{2}{\sqrt{\pi}}\left(x-\frac{x^{3}}{3}\right)$. Therefore, equation (4) can be approximated as

$$
\operatorname{MSE}(s, c) \approx-\ln \left(12 c \sqrt{s}-c^{3} \sqrt{s^{3}}\right)+\ln (12 \sqrt{\pi})
$$

\section{For 1/f Noise}

The average standard deviation of $n$ uncorrelated Gaussian variable $Y_{i}$

$$
\bar{\sigma}_{Y}=\frac{1}{n} \sum_{i}^{n}\left(\sigma_{Y i}\right)=\frac{1}{n} \sum_{i}^{n}\left(\sqrt{\lambda_{i}}\right)
$$

Each coarse-grained sequence with scale $s$, the data length becomes $\frac{n}{s}$. Therefore, the average standard deviation of the coarse-grained sequence becomes

$$
\bar{\sigma}_{Y S}=\frac{s}{n} \sum_{i}^{n / s}\left(\sigma_{Y i}\right)=\frac{s}{n} \sum_{i}^{n / s}\left(\sqrt{\lambda_{i}}\right)
$$

Li has reported that when the data length $n$ is sufficiently large, the eigenvalue of a fractal Brownian motion (fBm) process with Hurst exponent $H$ follows as a power-law[16]

$$
\lambda_{i} \approx \frac{K}{i^{2 H+1}}
$$

where $K$ is a constant related to $H$.

The power versus frequency relationship of $\mathrm{fBm}$ process is:

$|A(f)|^{2} \propto c_{0} \cdot f^{-\beta}$

where $\beta$ is the power law scaling factor. The Hurst exponent $H$ is related with $\beta$ when $\beta>1$ [14]: $H=(\beta-1) / 2$, therefore, equation (8) becomes

$$
\lambda_{i} \approx \frac{K}{i^{\beta}}
$$

Consider the partial sum,

$s_{n}=\sum_{k=1}^{n} a_{k}=1+\frac{1}{2^{p}}+\frac{1}{3^{p}}+\cdots+\frac{1}{n^{p}}$

Chlebus has given the lower bound of this partial sum for the case of $p=1$ as $\ln (n+1)$ [17].

From equation (9),

$n \sigma_{X}^{2}=n \sigma_{Y}^{2} \approx \sum_{i=1}^{n} \frac{K}{i^{\beta}} \approx K \ln (n+1)$

Therefore,

$K \approx \frac{n \sigma_{X}^{2}}{\ln (n+1)}$

Replace $K$ in equation (9)

$\lambda_{1} \approx \frac{K}{i^{\beta}} \approx \frac{n \sigma_{X}^{2}}{\ln (n+1)} \cdot \frac{1}{i^{\beta}}$

From equation (7) and (10), 


$$
\begin{aligned}
& \bar{\sigma}_{Y S}=\frac{s}{n} \sum_{i}^{n / s}\left(\sigma_{Y i}\right) \\
& =\frac{s}{n} \sum_{i}^{n / s}\left(\sqrt{\lambda_{i}}\right) \\
& \approx \frac{s}{n} \sum_{i}^{n / s}\left(\sqrt{\frac{n \sigma_{X}^{2}}{\ln (n+1)} \cdot \frac{1}{i^{\beta}}}\right) \\
& =\frac{s}{n} \frac{\sqrt{n} \sigma_{X}}{\sqrt{\ln (n+1)}} \cdot \sum_{i}^{n / s}\left(\frac{1}{i^{\beta / 2}}\right)
\end{aligned}
$$

For $1 / \mathrm{f}$ noise, $\beta=1$,

$\bar{\sigma}_{Y S} \approx \frac{s}{n} \frac{\sqrt{n} \sigma_{X}}{\sqrt{\ln (n+1)}} \cdot \sum_{i}^{n / s}\left(\frac{1}{i^{1 / 2}}\right)$

Using partial sum estimation of p-series in [18]

$$
\bar{\sigma}_{\mathrm{YS}} \approx \frac{s}{n} \frac{\sqrt{n} \sigma_{X}}{\sqrt{\ln (n+1)}} \cdot \frac{(n / s)^{1-\beta / 2}}{1-\beta / 2}=\frac{2 \sqrt{s} \sigma_{X}}{\sqrt{\ln (n+1)}}
$$

According to equation (12),

$$
\begin{aligned}
& E(s, \beta=1) \approx-\ln \left(\frac{2}{\sqrt{\pi}} \frac{\sqrt{s} c \sigma_{X}}{2 \bar{\sigma}_{Y}}\right) \\
& =-\ln \left(\frac{1}{\sqrt{\pi}} \frac{\sqrt{s} c \sigma_{X} \sqrt{\ln (n+1)}}{2 \sqrt{s} \sigma_{X}}\right) \\
& =-\ln \left(\frac{c \sqrt{\ln (n+1)}}{2 \sqrt{\pi}}\right)
\end{aligned}
$$

For a typical setting, $c=0.15, n=30000, E(s ; \beta=1) \approx 1.9961$.

If we keep the first two terms of $\operatorname{erf}(x)$, as $\operatorname{erf}(x)=\frac{2}{\sqrt{\pi}}\left(x-\frac{x^{3}}{3}\right)$. Therefore, equation (13) can be approximated as

$$
\begin{aligned}
& E(s, \beta=1) \\
& \approx-\ln \left\{\frac{c \sqrt{\ln (n+1)}}{2 \sqrt{\pi}}-\frac{c^{3}[\ln (n+1)]^{\frac{3}{2}}}{96 \sqrt{\pi}}\right\}
\end{aligned}
$$

\section{Evaluations}

In this section, the proposed analytical MSE, both for first order and second order expansion, are compared to the numerical MSE, and computed in a custom-made Matlab toolbox by Ahmed and Mandic [19]. The goodness of fit method (Matlab function GoodnessOfFit() ), defined as the normalized root mean square error, is used to evaluate how well the analytical MSE comparing to numerical MSE for simulated white noise and 1/f noise. The closer the value is to zero, the better the fit. All the numerical MSE are the means of 30 trials (where each trial has 10000 Gaussian noise data points) with the error bars indicating the standard deviations. The relative estimation error (REE) is defined as the absolute value of difference between the analytical MSE approximation and the original MSE divided by the original MSE.

\section{Evaluation of Analytical MSE for White Noise}

Uncorrelated Gaussian noise is generated by Matlab function randn. There are two parameters in equation (5) and (6), scale factor $s$ and tolerance factor $c$. Figure 1(a) and (b) display the analytical and numerical sample entropy against the tolerance factor $c$. Figure 1(c) and (d) show the MSE analysis with the scales in natural logarithm. First order and second order approximations are compared (Figure 1(a) vs (b); Figure 1(c) vs (d)).

We can observe that: (i) the analytical and numerical curves in each sub-figure are very similar, suggested by the small goodness-of-fit values; (ii) the second order analytical MSE approximations (Figure 1(b) and (d)) is better than the first order counterparts (Figure 1(c) and (d)), suggested by 
smaller fit values; (iii) the analytical solution performance decreases with tolerance factor $c$, suggested by higher REE for higher $c$, reaching maxima when $c=0.5$ (REE for first order: $1.74 \%$, for second order: $0.10 \%$ ); (iv) the analytical solution performance decreases with scale factor $s$, suggested by higher REE for higher $\ln s$, reaching maxima when $s=20$ (REE for first order:3.41\%, for second order: $0.38 \%$ ).

Generally, the low fit values show that the proposed analytical MSE formula is well fitted to the original MSE for white noise.

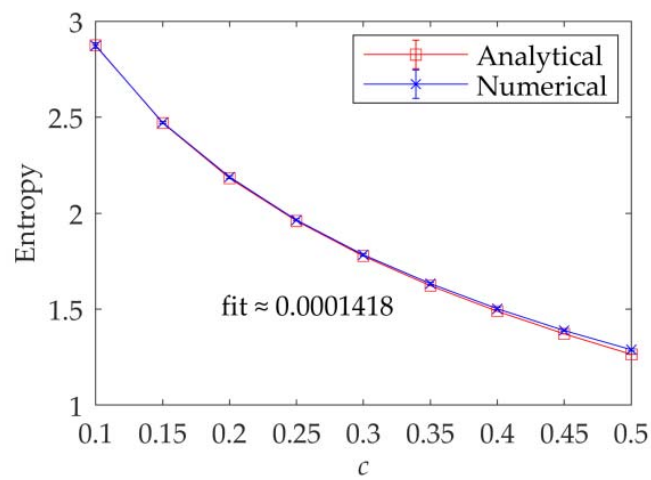

(a)

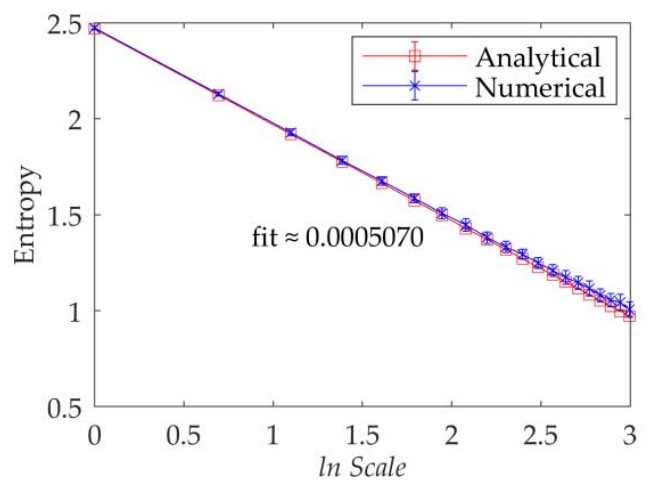

(c)

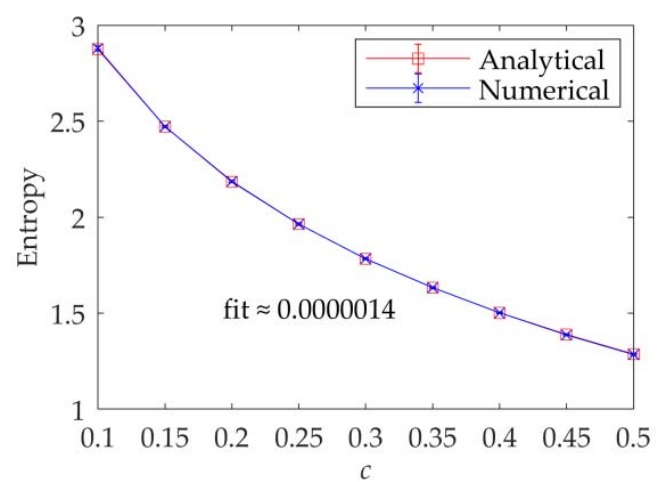

(b)

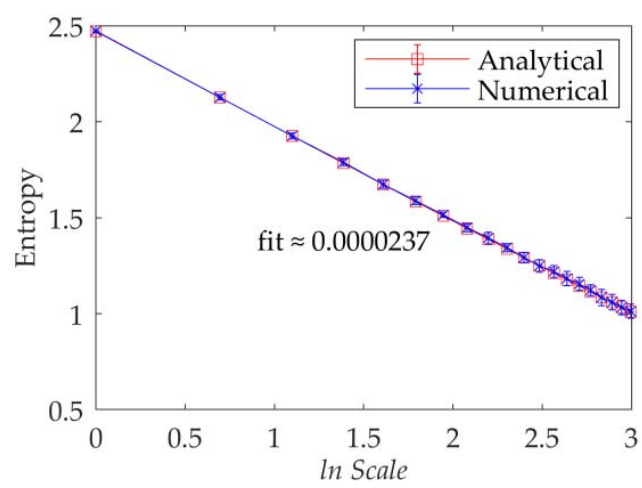

(d)

Figure 1. Analytical and numerical MSE comparison FOR White noise. (a) First order in each $c$ values while $s=1$. (b) Second order in each c values while $s=1$. (c) First order in each $\ln s$ values while $c=0.15$. (d) Second order in each $\ln s$ values while $c=0.15$.

\section{Evaluation of Analytical MSE for 1/f Noise}

There are two parameters in equation (13) and (14), tolerance factor $c$ and data length $n$. The analytical and numerical MSE values vs. $c$ are shown in Figure 4(a), for the first order expansion; and (b), for the second order. Set $c=0.15$, MSE values are plotted against the natural logarithms of scale factor s (Figure 4(c) for first order and (d) for second order). Goodness-of-fit values are plotted against data length $n$ (Figure 4(e)).

We can observe: (i) The analytical and numerical MSE curves are very similar in (a), (b), (c) and (d), and the small fit values show that the analytical and numerical MSE values fit well; (ii) the second order analytical MSE values seems not better than that in first order in terms of the goodnessof-fit values, yet a constant bias can be observed for the second order (Figure 2 (b)) in contrast with the intercepting curves (Figure 2 (a)); (iii) Data length $n$ has less impact on the analytical approximation of MSE when $n>10000$ (Figure 4(e)).

The low fit values show that the proposed analytical MSE formula is also well fitted to the original MSE for $1 / \mathrm{f}$ noise. 


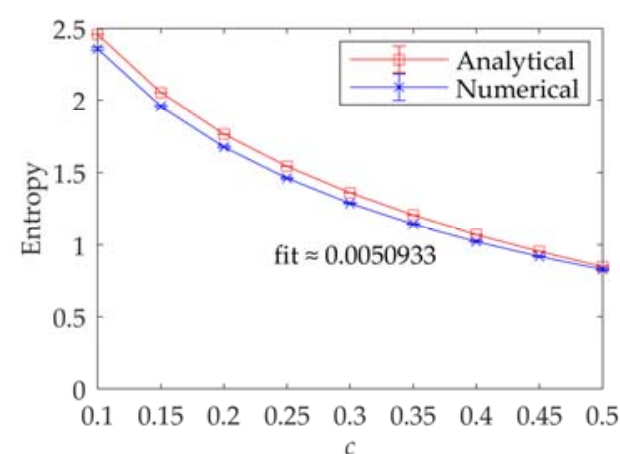

(a)

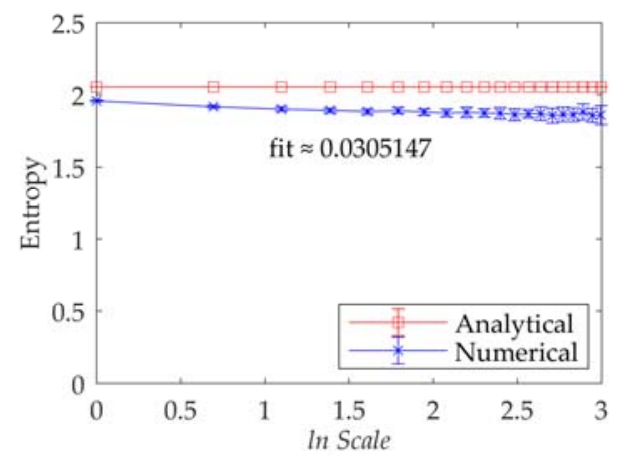

(c)

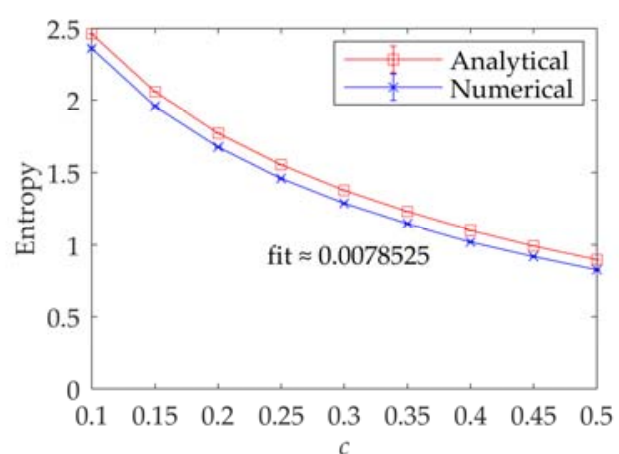

(b)

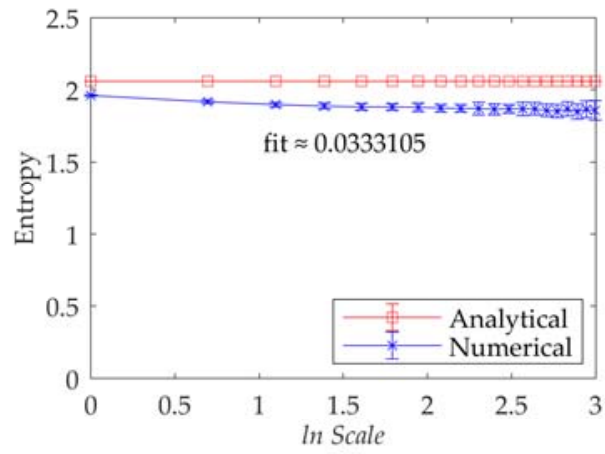

(d)

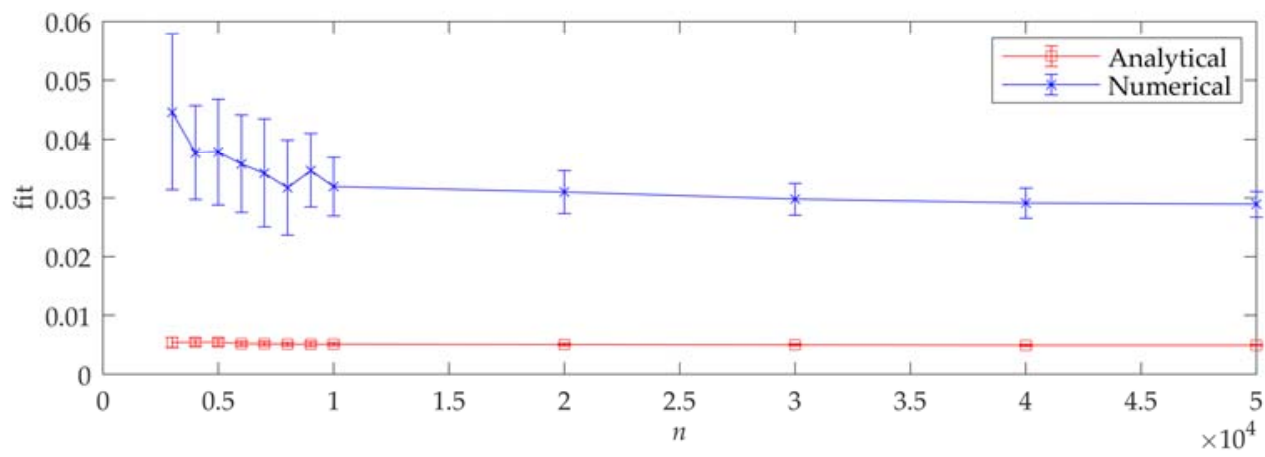

(e)

Figure 2. Analytical and numerical entropy comparison FOR $1 / f$ noise with $c=0.15$. Data length $n=10000$ for (a-d), $n=0$ 50000 for (e). (a) First order with $s=1$; (b) Second order with $s=1$; (c) First order with $c=0.15$; (d) Second order with $c=0.15$; (e) The goodness-of-fit values for (a) and (c) as functions of data length $n$; error bar indicating standard deviations.

\section{Discussions}

To better understand the MSE, we have proposed a new analytical solution for MSE. Reference [13] gives us the analytical formula (proportional relation) showing that despite the scaling factor, the tolerance factor is also important in separating different physiologic conditions. We have found the bias in that analytical formula and have provided more accurate formulae in this work.

The analytical scaling terms in the formulae are well fitted to the numerical results for both white noise and 1/f noise. First order expansion of Taylor series is adequate in terms of the goodness-offit values. The analytical formula for white noise in equation (5) shows that MSE' values depends only on the tolerance factor and does not depend on the data length. In contrast, the analytical formula for $1 / \mathrm{f}$ noise in equation (13) shows that MSE' values depend both on the tolerance factor and the data length.

Note that, the results we present in this work is an approximation with Taylor series expansion, $\mathrm{p}$ series expansion and Li's approximation, and can only serve a heuristic purpose. 


\section{Conclusions}

We have proposed a straightforward analytical solution of MSE for two special cases, white noise and 1/f noise. The proposed analytical solution may deepen the understanding of the widely applicated MSE method in the analysis of biological signals.

\section{Acknowledgment}

We thank Siqi Yang for valuable discussion. Han and Zhuo contributed equally. Fei is the cocorresponding author.

\section{References}

[1] S. M. Pincus, “Assessing serial irregularity and its implications for health," Annals of the New York Academy of Sciences, vol. 954, pp. 245-67, Dec, 2001.

[2] H. X. Zhang, Y. S. Zhu, and Z. M. Wang, "Complexity measure and complexity rate information based detection of ventricular tachycardia and fibrillation," Medical and Biological Engineering and Computing, vol. 38, no. 5, pp. 553-7, Sep, 2000.

[3] L. Quan, C. Yi-Feng, F. Shou-Zen, M. F. Abbod, S. J. C. Jiann-Shing, and Mathematical Methods in Medicine, "EEG Signals Analysis Using Multiscale Entropy for Depth of Anesthesia Monitoring during Surgery through Artificial Neural Networks,” vol. 2015, no. 1, pp. 53-57, 2015.

[4] M. Costa, A. L. Goldberger, and C. K. Peng, "Multiscale entropy analysis of complex physiologic time series,” Physical Review Letters vol. 89, no. 6, pp. 068102 , Aug 5, 2002.

[5] M. Costa, A. L. Goldberger, and C. K. Peng, "Multiscale entropy analysis of biological signals," Physical Review. E: Statistical, Nonlinear, and Soft Matter Physics, vol. 71, no. 2 Pt 1, pp. 021906, Feb, 2005.

[6] F. Liao, G. L. Y. Cheing, W. Ren, S. Jain, and Y. Jan, “Application of Multiscale Entropy in Assessing Plantar Skin Blood Flow Dynamics in Diabetics with Peripheral Neuropathy,” Entropy, vol. 20, no. 2, pp. 127, 2018.

[7] R. Okazaki, T. Takahashi, K. Ueno, K. Takahashi, M. Ishitobi, M. Kikuchi, M. Higashima, and Y. Wada, "Changes in EEG Complexity with Electroconvulsive Therapy in a Patient with Autism Spectrum Disorders: A Multiscale Entropy Approach,” Frontiers in Human Neuroscience, vol. 9, pp. 106-106, 2015.

[8] X. Chen, Q. Guo, and D. G. Brockway, "Analyzing the complexity of cone production in longleaf pine by multiscale entropy,” Journal of Sustainable Forestry vol. 35, no. 2, pp. 172-182, 2016.

[9] H. Niu, and J. Wang, "Quantifying complexity of financial short-term time series by composite multiscale entropy measure," Communications in Nonlinear Science and Numerical Simulation, vol. 22, no. 1, pp. 375-382, 2015.

[10] L. Guzmanvargas, A. Ramirezrojas, and F. Angulobrown, "Multiscale entropy analysis of electroseismic time series,” Natural Hazards and Earth System Sciences, vol. 8, no. 4, pp. 855-860, 2008.

[11] Y. Yin, and P. Shang, "Multivariate multiscale sample entropy of traffic time series," Nonlinear Dynamics, vol. 86, no. 1, pp. 479-488, 2016.

[12] M. Barner, C. Cottineau, C. Molinero, H. Salat, K. Stanilov, and E. Arcaute, "Multiscale Entropy in the Spatial Context of Cities,” Physics 2017. 
[13] J. Gao, J. Hu, F. Liu, and Y. Cao, "Multiscale entropy analysis of biological signals: a fundamental bi-scaling law,” Frontiers in computational neuroscience, vol. 9, pp. 64, 2015.

[14] A. Eke, P. Herman, J. B. Bassingthwaighte, G. M. Raymond, D. B. Percival, M. Cannon, I. Balla, and C. Ikrenyi, "Physiological time series: distinguishing fractal noises from motions," Pflügers Archiv: European Journal of Physiology, vol. 439, no. 4, pp. 403-15, Feb, 2000.

[15] K. Briggs, "Integrals involving erf," http://keithbriggs.info/documents/erf-integrals.pdf, 2003].

[16] L. Li, J. Hu, Y. Chen, and Y. Zhang, "PCA based Hurst exponent estimator for fBm signals under disturbances,” Signal Processing, IEEE Transactions on, vol. 57, no. 7, pp. 2840-2846, 2009.

[17] E. Chlebus, “An approximate formula for a partial sum of the divergent p-series," Applied Mathematics Letters, vol. 22, no. 5, pp. 732-737, 2009.

[18] S. K. Goel, and D. M. Rodriguez, "A note on evaluating limits using Riemann sums," Mathematics Magazine, pp. 225-228, 1987.

[19] M. U. Ahmed, and D. P. Mandic, "Multivariate multiscale entropy: A tool for complexity analysis of multichannel data,” Physical Review. E: Statistical, Nonlinear, and Soft Matter Physics, vol. 84, no. 6, pp. 061918, 2011. 